\title{
Wavelength-locking of a Semiconductor Laser using an Electronic Technique
}

\author{
K. Mullaney, J. Hodgkinson*, S. E. Staines and R. P. Tatam \\ Engineering Photonics, Cranfield University, Bedfordshire, MK43 0AL, UK.
}

\begin{abstract}
This work describes a novel system to control the stability of a $1583 \mathrm{~nm}$ telecommunications laser diode via measurement of junction voltage. This electronic technique dispenses with the optical components used in conventional wavelength locking schemes and shifts wavelength control to system level electronic instrumentation. The approach employs real-time measurement of diode series resistance $\left(R_{s}\right)$, which is used to compensate the measured forward voltage $\left(V_{f}\right)$ and recover the junction voltage $\left(\mathrm{V}_{\mathrm{j}}\right)$ of the laser. Control of $\mathrm{V}_{\mathrm{j}}$ provides wavelength control without introducing a significant error when the package temperature varies. This was implemented by measuring Rs as the dynamic resistance, $\delta \mathrm{V} / \delta \mathrm{I}$, by modulating the injection current. Recent work has reduced the modulation amplitude and noise in the electronics. Using a frequency deviation of $1 \mathrm{GHz}$, we achieved a centre wavelength variation of $\pm 2 \mathrm{pm}$ over a package temperature variation of $20-55^{\circ} \mathrm{C}$. This gives a wavelength/ temperature coefficient of $0.03 \mathrm{pm} /{ }^{\circ} \mathrm{C}$, which is an improvement on $0.34 \mathrm{pm} /{ }^{\circ} \mathrm{C}$, as typically achieved for optical locking systems. The system has been further developed using board-level components within a compact demonstrator unit. Work is on-going to further enhance this performance over a package temperature variation of $0-70{ }^{\circ} \mathrm{C}$.
\end{abstract}

Keywords: DFB laser, wavelength locking, telecommunications, LED, frequency, electronic.

\section{INTRODUCTION}

The wavelength stability of laser diodes underpins many applications in photonics. Typical applications are telecommunications and gas spectroscopy which have stringent requirements for both short term and long term operation. Telecommunications using dense wavelength division multiplexing (DWDM), restricts lasers to operation within the International Telecommunication Union (ITU) grid ${ }^{[1]}$, operating in the range $1530-1625 \mathrm{~nm}$ with a channel spacing ranging from $10 \mathrm{GHz}$ to $100 \mathrm{GHz}$, centered with a frequency precision of $\Delta v= \pm 1.5 \mathrm{GHz}(\Delta \lambda= \pm 12 \mathrm{pm})^{[2]}$. In high resolution gas spectroscopy, a laser's emission wavelength is scanned across a narrow gas absorption line to measure the gas concentration ${ }^{[3]}$. The wavelength drift of the laser must be much smaller than the gas linewidth, which for methane at $\lambda=1651 \mathrm{~nm}$ is $\Delta \lambda=40 \mathrm{pm}$ full width half maximum ${ }^{[3]}$.

For these applications, laser diodes such as distributed feedback (DFB) lasers are used, with a linewidth of around $\Delta v=20$ $\mathrm{MHz}$ or lower ${ }^{[4]}$. Although lasers are nominally produced at a given wavelength to a tolerance of perhaps $\Delta \lambda=0.1 \mathrm{~nm}$ ) ${ }^{[4]}$, the precise emission wavelength is a function of the injection current and laser operating temperature. The latter is affected by the external package (case) temperature, junction heating and thermal gradients within the package. Even with good thermal package design, active wavelength stabilization is required ${ }^{[5,6]}$. If the injection current is fixed and the temperature of the laser's active region can be precisely and accurately controlled, the emitted wavelength should remain constant.

Conventional temperature control employs a thermo-electric cooler (TEC) and thermistor, the latter being located typically several millimeters from the laser's active region. This creates systematic errors ${ }^{[5]}$ as the control system measures the temperature of the thermistor rather than the laser, thus is affected by changes to the temperature gradient between the thermistor and the active region, caused by changes to the external (case) temperature over the specified working temperature range.

*j.hodgkinson@cranfield.ac.uk; phone +44 1234758325 
To improve the wavelength stability provided by thermistor control a number of optical techniques are currently used. Thermally stable optical filters and etalons typically offer $\sim 1 \mathrm{GHz}$ stability over the life and operating temperature range of the device ${ }^{[7]}$ and are used in wavelength lockers. These lockers can either be either internal or external to the package, as a separate device. These techniques inevitably increase the package component count (collimating optics, etalon, beam splitter, PIN diodes) and require active optical alignment during assembly to ensure the laser output is aligned to the ITU grid. Gas reference cells are also often used as wavelength transfer standards in telecoms applications ${ }^{[8]}$.

An alternative control technique is to use the electrical properties of the laser diode, such as its forward voltage $\mathrm{V}_{\mathrm{f}}$, as this provides a sensitive indicator of the junction temperature. Potential advantages of this latter approach are a significant reduction in component count within the package and a reduced number of optical alignment processes during assembly.

Uehara and Katakura proposed the use of $\mathrm{V}_{\mathrm{f}}$ to stabilize the temperature and wavelength of a laser diode, and applied this to a $1.3 \mu \mathrm{m}$ buried heterostructure distributed feedback (BH DFB) laser ${ }^{[9]}$. They were able to achieve a stability of $\Delta \lambda=2$ pm over 30 minutes at a fixed temperature, and a systematic shift of $\Delta \lambda=3 \mathrm{pm}$ when subject to an external change in the ambient temperature of $10^{\circ} \mathrm{C}$. Lin et al. have applied forward voltage based control to a Fabry Perot laser diode ${ }^{[10]}$. They proposed that the injection current affects the junction temperature and that changes to this parameter must be compensated for when using the forward voltage. The forward voltage has also been used by Sergachev et al. in a fast $(300 \mathrm{kHz}$ bandwidth) control loop aimed at reducing frequency noise in quantum cascade lasers ${ }^{[11]}$.

In this paper we report on the progress in developing a new wavelength stabilization technique, whereby the junction voltage $\left(\mathrm{V}_{\mathrm{j}}\right)$ of a laser diode is used to measure the temperature of the active medium. The essence of the technique is that the series resistance of the laser diode can impose a small but significant error when measuring the forward voltage $V_{f}$, and must be compensated to recover, accurately, the underlying junction voltage ${ }^{[5]}$. Applying a sinusoidal modulation to the injection current and measuring the demodulated forward voltage allows one to measure the series resistance dynamically as $\delta \mathrm{V} / \delta \mathrm{I}$ and compensate for it. Modulation of the injection current also has the effect of frequency modulating the laser output.

This paper further develops the technique which was described by Asmari et al. and applied to a 1650nm DFB laser packaged in a TO can ${ }^{[5]}$. We have now reapplied this technique to a telecommunications DFB fiber-coupled laser and replicated the results. The frequency modulation of the laser output has been reduced from $40 \mathrm{pm}$ to $9 \mathrm{pm} \mathrm{pk}$. to pk. and the temperature range over which stability was achieved has been increased from $20{ }^{\circ} \mathrm{C}$ to $35^{\circ} \mathrm{C}$. The experimental set-up has also been improved; migrating from discrete laboratory instruments to a compact rack-mountable unit comprising discrete boards/modules.

\section{LOCKING METHOD}

The relationship between a laser diode's junction voltage $\left(\mathrm{V}_{\mathrm{j}}\right)$ and its operating temperature can be used as a temperature sensor and, within a control loop, to stabilise the centre wavelength of the laser diode ${ }^{[5]}$. For DFB laser diodes requiring injection currents $\left(\mathrm{I}_{\mathrm{f}}\right)$ of the order of $155 \mathrm{~mA}$, changes in series resistance $\left(\mathrm{R}_{\mathrm{s}}\right)$ with temperature can create a small but significant systematic error ${ }^{[5]}$ in the measured $V_{\mathrm{f}}$. The simplified relationship between these terms is described in the equation below.

$$
V_{f}=V_{j}+I_{f} R_{s}
$$

The methodology of junction voltage based temperature control is as follows. The $\mathrm{V}_{\mathrm{f}}$ of the laser diode is measured during typical operation. Then a small, $\mathrm{kHz}$ sinusoidal signal, $\mathrm{I}_{\mathrm{f}}$, is applied to the laser resulting in a small modulation of both the wavelength and the forward voltage. The magnitude of the modulated voltage is measured and gives a dynamic measure of the diode series resistance as $R_{s}=\delta V_{f} / \delta I$. This value of $I_{f} R_{s}$ is subtracted from $V_{f}$ and provides the underlying junction voltage $\mathrm{V}_{\mathrm{j}}$ which has been corrected for dynamic temperature effects. In practical terms, this is achieved by scaling the measured modulation $\delta V_{f}$ using a scaling constant $A_{c}$, where $A_{c}=I_{f} / \delta I$. The scaling constant is then subtracted from the measured DC value of $V_{f}$. The junction voltage is then subtracted from a selected set-point 
voltage and the resulting set-point error is fed into a PID loop that controls the current to the TEC. A flow diagram for the process is given in Figure 1.

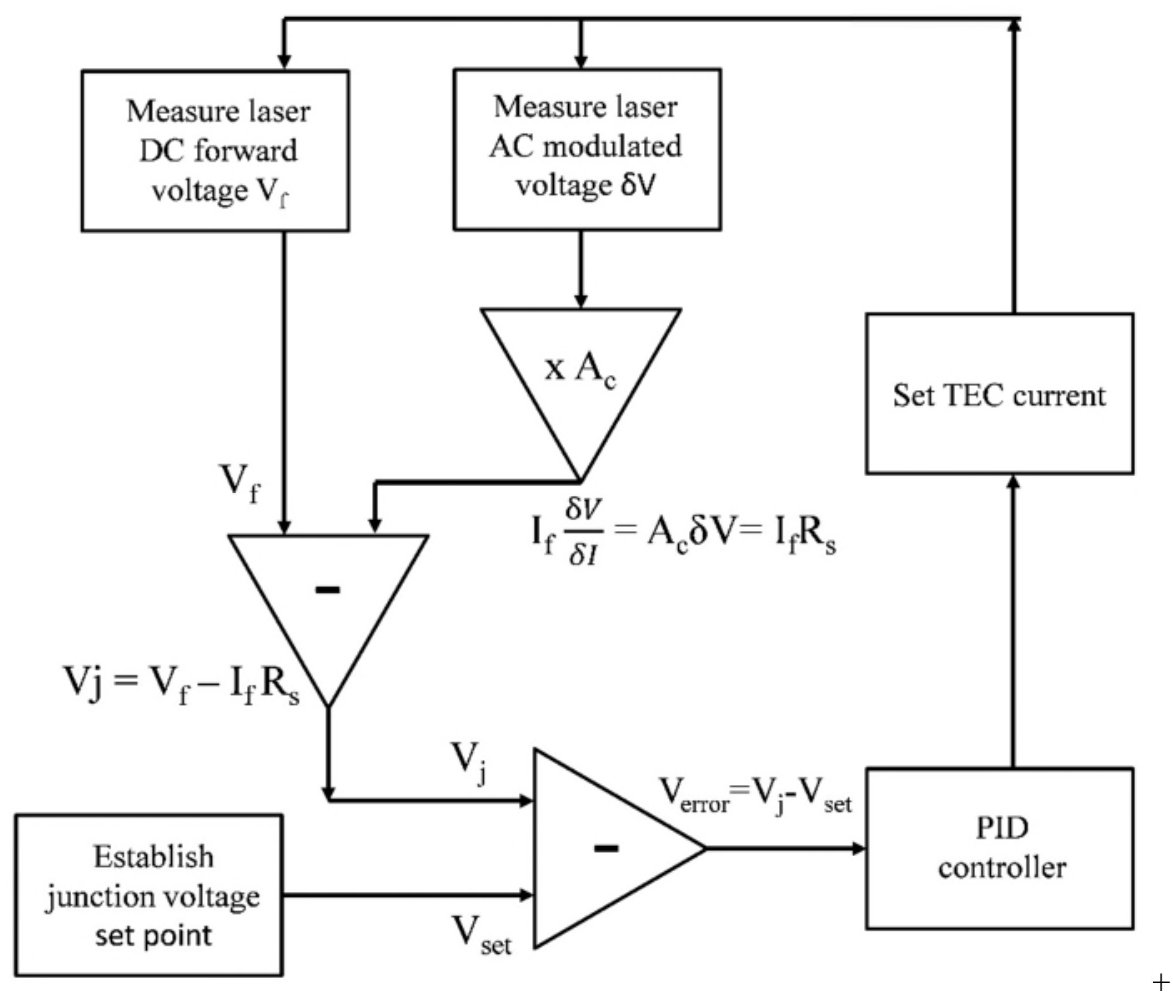

Figure 1. Method for junction voltage laser wavelength control, utilizing forward voltage with correction for series resistance.

\section{EXPERIMENTAL METHOD}

\subsection{Initial experimental arrangement}

The experimental arrangement for wavelength control using junction voltage is shown in Figure 2. For this work, a 1583 $\mathrm{nm}$ InGaAsP DFB telecoms laser (Nortel Networks, LC155CDC-20) was used as the light source. This device has an integrated optical wavelength locker and thermistor for wavelength control. This device was selected as it is representative of DFBs used in telecommunications networks, and from a package perspective, it is designed to exhibit high stability over a wide temperature range. A current driver (Thorlabs LDC200) was used to bias the laser with a fixed DC injection current of $155 \mathrm{~mA}$ which produced an output power of $12 \mathrm{~mW}$. In addition to the DC bias, the laser diode was modulated at 26 $\mathrm{kHz}$ with a current amplitude of $1.3 \mathrm{~mA}$ pk-pk using a function generator (Rigol DG4162). This resulted in a wavelength modulation of $9 \pm 2 \mathrm{pm}$ at $-3 \mathrm{~dB}$. A lock-in amplifier (Stanford Research SR850) was used to measure the $V_{\mathrm{f}}$ at the first harmonic of the modulation frequency. The voltage output from the lock-in was passed to a National Instruments data acquisition card (PCI 6259) and used to compensate the $\mathrm{V}_{\mathrm{f}}$ in order to recover the junction voltage. The scaling constant $\left(\mathrm{A}_{\mathrm{c}}=\mathrm{I}_{\mathrm{f}} / \delta \mathrm{I}\right)$ used for this compensation was determined using a starting value of $2.6 \Omega$ for $\mathrm{R}_{\mathrm{s}}{ }^{[5]}$ and then by making iterative changes, until the controller was stable. Again, voltage compensation and the PID controller were implemented in LabVIEW. 


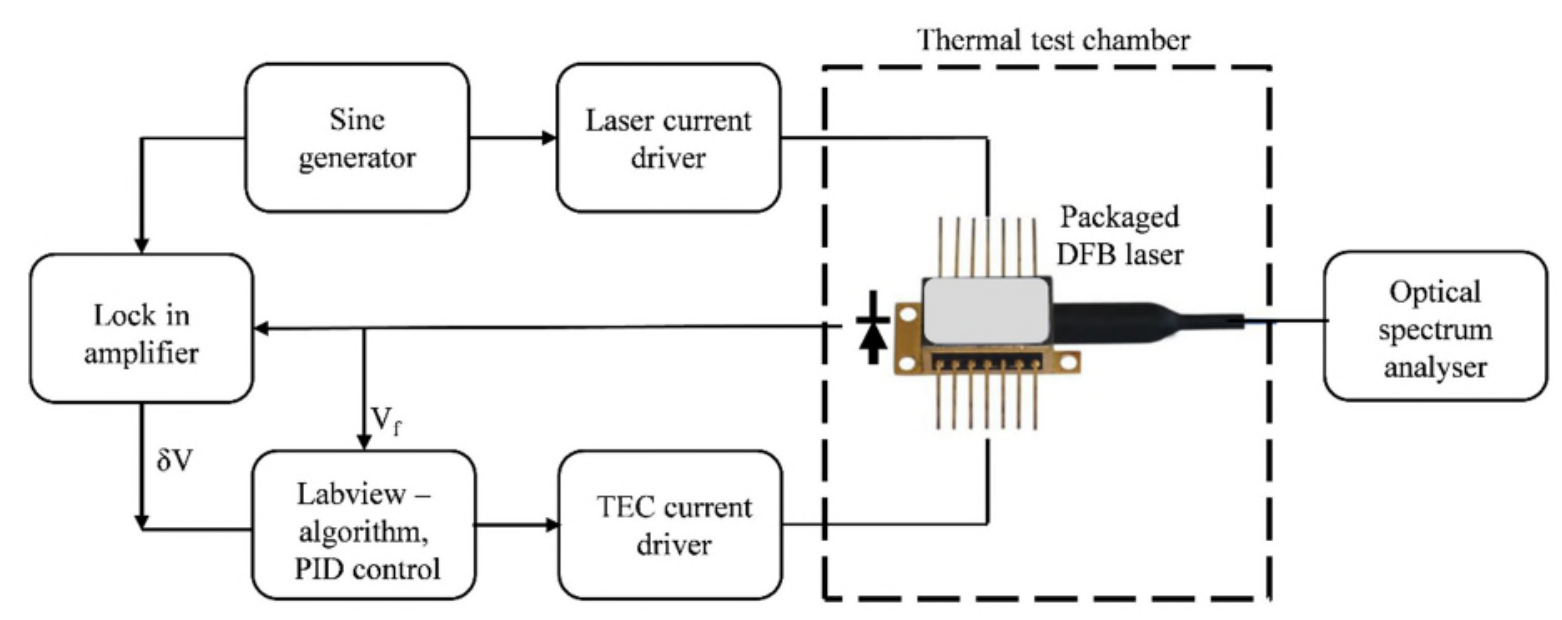

Figure 2. Experimental arrangement for wavelength locking a DFB laser using the junction voltage technique.

The output from the PID controller was connected to the modulation input of a second current driver (Thorlabs ITC510) in order to close the feedback loop to the thermo-electric cooler (TEC) in the laser. The laser was located in a butterfly mount (Thorlabs LM14S2) which in turn was placed in a thermal-cycling chamber (Votsch VTM7004). Connecting cables were passed through ports in the side of this chamber to the instruments described previously. The thermal chamber allows the case temperature of the laser to be varied from 0-70 ${ }^{\circ} \mathrm{C}$ reproducibly, and hence assess the impact on wavelength stability. An optical spectrum analyzer (OSA) (Yokogawa AQ6370C-10) and wavelength meter (Advantest TQ8323, repeatability $\pm 1 \mathrm{pm}$ ) were used to assess the stability of the laser.

\subsection{Experimental results}

\section{Thermistor wavelength control}

To benchmark the wavelength stability of the laser diode it was evaluated while it was under thermistor control. The internal thermistor is typically mounted on the laser tile adjacent to the laser chip and the wavelength locker optical components. The laser was driven by a Thorlabs LDC200 and the TEC and thermistor were controlled by a Thorlabs TED200 temperature controller. The butterfly package was placed inside the thermal chamber and the temperature was varied in six steps between $0-70{ }^{\circ} \mathrm{C}$ allowing the package to thermal stabilize for 30 minutes at each step, while the internal thermistor was set to $20{ }^{\circ} \mathrm{C}$. Over $0-70{ }^{\circ} \mathrm{C}$ there was a $21 \mathrm{pm}$ wavelength variation equating to a systematic wavelength change of $0.3 \mathrm{pm} /{ }^{0} \mathrm{C}$.

\section{Junction voltage control}

Using the system described in section 3.1, the scaling constant $\left(\mathrm{A}_{\mathrm{c}}\right)$ was adjusted while the wavelength deviation was measured over the laser case temperature range of $20-55{ }^{\circ} \mathrm{C}$, and the $\mathrm{A}_{\mathrm{c}}$ was identified which gave minimum wavelength deviation. Typical measured results are shown in Table 1 . As the case temperature rises, the $V_{F}$ increase is compensated by an increase in $I_{f} R_{s}$ due to the series resistance of the diode increasing. Accordingly, the junction voltage $\left(V_{J}\right)$ remains constant at $\mathrm{V}_{\mathrm{SET}}=1.35$ due to the temperature increase and therefore maintains good wavelength stability.

Over the temperature range $20-55{ }^{\circ} \mathrm{C}$ the wavelength stability of the center wavelength was typically \pm 2 pm when the PID controller was adjusted to $\mathrm{V}_{\mathrm{SET}}=1.35$. This result was achieved while the output wavelength was modulated at $9 \pm 2 \mathrm{pm}$. Over $20-55{ }^{\circ} \mathrm{C}$ there was a $1 \mathrm{pm}$ wavelength variation in centre wavelength equating to a wavelength stability of $0.03 \mathrm{pm} /$ ${ }^{0} \mathrm{C}$. 
Table 1. Typical wavelength stability of a DFB laser diode under junction voltage wavelength control.

\begin{tabular}{|c|c|c|c|c|c|c|c|}
\hline Case temp. $/{ }^{\mathbf{0}} \mathbf{C}$ & $\begin{array}{c}\text { Laser output } \\
\boldsymbol{\lambda} / \mathbf{n m}\end{array}$ & $\begin{array}{c}\boldsymbol{\lambda} \text { change } \\
/ \mathbf{p m}\end{array}$ & $\mathbf{I}_{\mathbf{f}} \mathbf{R}_{\mathbf{s}} / \mathbf{V}$ & $\mathbf{V}_{\mathbf{F}} / \mathbf{V}$ & $\mathbf{V}_{\text {SET }} / \mathbf{V}$ & $\begin{array}{c}\mathbf{T E C} \text { current } / \\
\mathbf{m A}\end{array}$ & Ac \\
\hline 20 & 1583.015 & 0 & 0.3842 & 1.734 & 1.35 & 137 & 9.65 \\
\hline 55 & 1583.014 & -1 & 0.387 & 1.737 & 1.35 & 703 & 9.65 \\
\hline
\end{tabular}

\subsection{Integrated system}

The laboratory instruments described in the previous section were replaced with a compact system with improved noise performance. The initial experimental system was susceptible to mains borne interference such that the variation on the $\mathrm{V}_{\mathrm{j}}$ signal line was $\pm 2 \mathrm{mV}$, whereas the new system had a maximum variation of $\pm 0.2 \mathrm{mV}$.

Commercially available components were used in the new system. A single-board dual phase lock-in amplifier (Femto LIA-BVD-150) replaced the Stanford SR850. This amplifier is supplied with an internal sine oscillator $5 \mathrm{~Hz}-130 \mathrm{kHz}$ which both supplies the reference signal and the modulation signal to the laser diode driver. A National Instruments USB 6212 multifunction DAQ, 16 bit resolution, sample rate $400 \mathrm{kSs}^{-1}$ replaced the DAQ card in the PC. The DAQ was now controlled via a USB interface to an external computer.

Two compact laser drivers were used to power the laser in the new system. To drive the TEC, an Arroyo instruments laser driver $2 \mathrm{~A}$ ( $65 \mathrm{kHz}$ modulation bandwidth) was used. This unit was controlled via a USB interface. The laser was driven by a Thorlabs IP500 $500 \mathrm{~mA}$ driver which has a $50 \mathrm{kHz}$ modulation bandwidth. The noise and ripple of this driver is $<10$ $\mu \mathrm{A}$ r.m.s. The current through the laser diode was fixed at $155 \mathrm{~mA}$. The arrangement of the components is shown in Figure 3. The case is divided into two screened compartments with power supplies, cooling fan and mains filter (Schaffner 0-400 $\mathrm{Hz}$ EMI filter) on the right hand side and the signal processing boards on the left. This arrangement helped to significantly reduce the mains driven noise level compared to the early experimental arrangement. As previously described the laser was located in a mount which was placed in the thermal-cycling chamber. The control laptop was connected via USB connectors at the rear of the case. Work is on-going to characterize and optimize the frequency stability of this system.

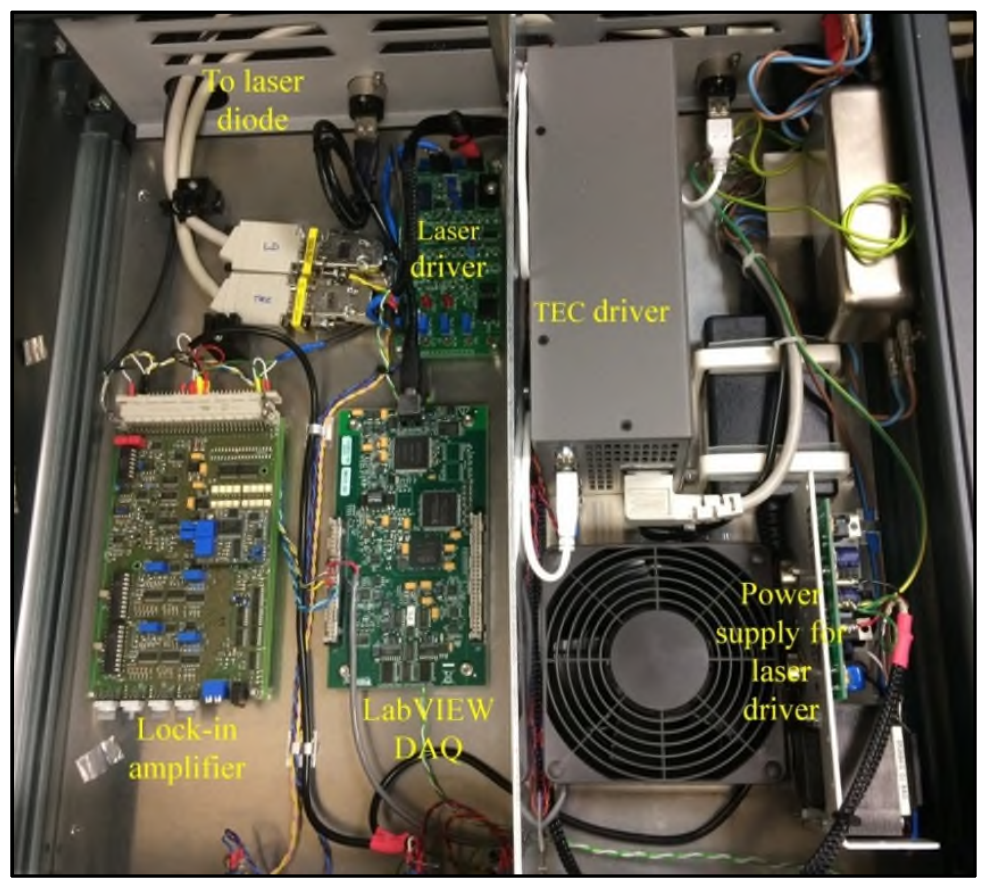

Figure 3. Internal view of the integrated system for locking a DFB laser using the junction voltage technique. 


\section{CONCLUSIONS}

In summary, the relationship between a laser diode's junction voltage and its operating temperature can be used as a temperature sensor and, within a control loop, to stabilize the central wavelength of the laser diode. For DFB laser diodes requiring injection currents of the order of $155 \mathrm{~mA}$, changes in the small series resistance with temperature can create a small but significant systematic error in the measured forward voltage. The technique described in this work uses this effect to correct the measurement of junction voltage so allowing it to be used as an accurate wavelength locking method. The latest results achieved from the experimental work are shown in Table 2 and compare favorably with the stability provided by current optical locking schemes $\left(0.34 \mathrm{pm} /{ }^{0} \mathrm{C}\right){ }^{[7]}$ used in telecommunications systems.

Table 2 . Wavelength stability of the junction voltage technique.

\begin{tabular}{|c|c|}
\hline Control technique & $\begin{array}{c}\text { Wavelength stability with case } \\
\text { temp. variation: } \mathrm{pm} /{ }^{0} \mathrm{C} .\end{array}$ \\
\hline Thermistor & $0.3 \mathrm{pm} /{ }^{0} \mathrm{C}$ \\
\hline $\begin{array}{l}\text { Junction voltage } \mathrm{Vj}- \\
\text { experimental system }\end{array}$ & $0.03 \mathrm{pm} /{ }^{0} \mathrm{C}$ \\
\hline
\end{tabular}

In future work, the electronic design could be simplified and substantially miniaturized so that it could be embedded into laser diode controllers. The existing wavelength modulation could also be minimized to a level at or below the residual wavelength fluctuation noise. This technique might also usefully be applied to a wide range of active optical emitters e.g. LEDs, quantum cascade lasers and tunable lasers.

\section{ACKNOWLEDGEMENTS}

This project was funded by the UK Engineering and Physical Sciences Research Council (EPSRC grants EP/K503927 and EP/N002520).

\section{REFERENCES}

[1] International Telecommunication Union, Recommendation ITU-T G.694.1 Spectral grids for WDM applications: DWDM frequency grid. ITU, Geneva, Switzerland (2012).

[2] Roppelt M., Grobe K., Eiselt M., and Elbers J.-P., "Investigation of wavelength control schemes in WDM-PONs", Paper 29, Proc ITG Symposium on Photonic Networks, (2011).

[3] Hodgkinson J. and Tatam R.P., “Optical gas sensing: a review”, Meas. Sci. Technol. 24, 012004 (59pp), (2013).

[4] Nanoplus, "Summary of specifications for DFB laser diodes from $1450 \mathrm{~nm}$ to $1650 \mathrm{~nm}$ ". Nanoplus Nanosystems and Technologies GmbH, Gerbrunn, Germany.

[5] Asmari A., Hodgkinson J., Chehura E., Staines S., and Tatam R.P., “All-electronic frequency stabilization of a DFB laser diode". Opt. Express, 25, 11679-11691 (2017).

[6] Vezzoni E., Gambini P. and Puleo M., "Laser frequency stabilization techniques for optical communication," Proc. 16th European Conference on Optical Communication (ECOC), 943-950 (1990). 
[7] Ackerman D. A., Paget K. M., Schneemeyer L. F., Ketelsen L. J.-P., Warning F. W., Sjolund O. , J. E. Graebner, Kanan A., Raju V. R., Eng L. E, Schaeffer E. D. and P. van Emmerik, "Low-cost athermal wavelength-locker integrated temperature-tuned single-frequency laser package," J. Lightwave Technol., 22(1), 166-171 (2004).

[8] Gilbert S. L., Swann W. C. and Dennis T., "Wavelength standards for optical communications," Proc. SPIE 4269, 184-191 (2001).

[9] Uehara K. and Katakura K., "New method of frequency stabilization of semiconductor lasers," Japanese J. Appl. Physics, 27(2), 244-246 (1988).

[10] Lin Z.-Q., Zhang Y., Lang Y.-H. and Yin F.-C., "Constant temperature control of semiconductor laser based on temperature characteristics of pn junction," Faguang Xuebao 30(2), 223-227 (2009).

[11] Sergachev I., Maulini R., Bismuto A., Blaser S., Gresch T., Bidaux Y., Müller A., Schilt S. and Südmeyer T., "All-electrical frequency noise reduction and linewidth narrowing in quantum cascade lasers," Opt. Lett. 39(22), 6411-6414 (2014). 
2019-03-04

\section{Wavelength-locking of a semiconductor laser using an electronic technique}

Mullaney, Kevin

SPIE

Mullaney K, Hodgkinson J, Staines SE, Tatam RPO. (2019) Wavelength-locking of a semiconductor laser using an electronic technique. In: SPIE OPTO: The Optoelectronics, Photonic Materials and Devices Conference, 2019, San Francisco, CA, USA, 2-7 February 2019, Proceedings Volume 10925, Photonic Instrumentation Engineering VI

https://doi.org/10.1117/12.2507260

Downloaded from Cranfield Library Services E-Repository 